\title{
Validated scattering kernels for triphenylmethane at cryogenic temperatures
}

\author{
Florencia Cantargi ${ }^{1, *}$, Javier Dawidowski ${ }^{1}$, Christian Helman ${ }^{1}$, José Ignacio Márquez Damian ${ }^{1}$, Jose Rolando Granada ${ }^{1}$, \\ Giovanni Romanelli ${ }^{2}$, Gabriel Julio Cuello ${ }^{3}$, Goran Skoro $^{2}$, and Matthew Krzystyniak ${ }^{2}$ \\ ${ }^{1}$ Neutron Physics Department, Centro Atómico Bariloche - Comisión Nacional de Energía Atómica, Argentina \\ ${ }^{2}$ ISIS Facility, Rutherford Appleton Laboratory, Chilton, Didcot, Oxfordshire OX11 0QX, United Kingdom \\ ${ }^{3}$ Institut Laue Langevin, 71, Av. des Martyrs, 38042 Grenoble, France
}

\begin{abstract}
Cold neutrons are widely used in different fields of research such as the study of the structure and dynamics of solids and liquids, the investigation of magnetic materials, biological systems, polymer science, and a rapidly growing area of industrial applications. In a pulsed neutron source where the pulse width is an important parameter to be considered, hydrogenated materials are often used because of their high energy transfer in each collision. The preliminary scattering kernel for triphenylmethane, a material of great potential interest for cold neutron production, had been presented at the ND2016 conference. Here, a new model for the generation of the scattering kernels for this material, together with experimental results on its total cross section measured at the VESUVIO instrument (ISIS Neutron and Muon Source, United Kingdom) is presented. The thermal scattering kernel was generated by means of the NJOY Nuclear Data Processing system, using as input the vibrational modes obtained by density functional theory techniques (DFT). The agreement between measurements and our model validates the scattering kernel construction and the cross section library generated in ENDF and ACE formats.
\end{abstract}

\section{Introduction}

The development and optimization of advanced cold neutron sources requires neutronic calculations involving thermal and sub-thermal neutron energies, which in turn demand the knowledge of reliable cross section data relative to the materials which form the system under consideration.

Condensed molecular systems often display a complex behavior due to translational, rotational and vibrational degrees of freedom - and their couplings - that animate the intra and inter-molecular motions. One of the main research lines at the Neutron Physics Department at Centro Atómico Bariloche is the development of scattering kernels for a number of molecular systems of interest, particularly cold moderator materials. A group of hydrogenous methylated aromatics such as benzene, toluene, mesitylene and mixtures of those were studied in the past [1].

The structural and dynamical properties of a given system determine the characteristics of its interaction with slow-neutrons, that is, the probabilities for the exchange of certain energy and momentum between the neutron and the target nucleus, as contained in the scattering law $S(\mathbf{Q}, \omega)$ of the system [2]. In the frame of the Gaussian approximation the dynamics of the material is enclosed in its generalized frequency spectrum $\rho$, and this is in fact the important piece of information we need to predict scattering probabilities in the case of hydrogenous materials, where interference effects are negligible.

*e-mail: cantargi@cab.cnea.gov.ar
Another group of potential moderator materials is now being studied. Among those, thiphenylmethane $\left(\mathrm{C}_{19} \mathrm{H}_{16}\right)$ had been proposed as a potentially good cold moderator due to its high protonic density and good radiation resistance [3]. This molecular compound is formed by three phenyl groups connected through a central carbon atom, being a colorless solid at room temperature.

The main goal of this work is the generation of a thermal cross section library for triphenylmethane at a low temperature, and its validation with experimental data obtained at the VESUVIO instrument (ISIS Neutron and Muon Source, Rutherford Appleton Laboratory).

\section{Thermal scattering law libraries generation}

As it was already mentioned, the calculation of $S(\mathbf{Q}, \omega)$ involves the characterization of its dynamics through a generalized frequency spectrum $\rho(\varepsilon)$ which can be obtained from experimental data, from theoretical models, numerical simulations, or a combination of those [4]. Once $\rho(\varepsilon)$ has been established, it is used to feed the LEAPR module of the NJOY nuclear data processing system [5], which employs a phonon expansion and the incoherent approximation to generate the inelastic cross section. This procedure is able to produce fairly accurate results in the case of hydrogeneous materials, where the incoherent contributions dominate the cross sections [6].

Nowadays, density functional theory (DFT) techniques are the state of start to obtain vibrational modes 


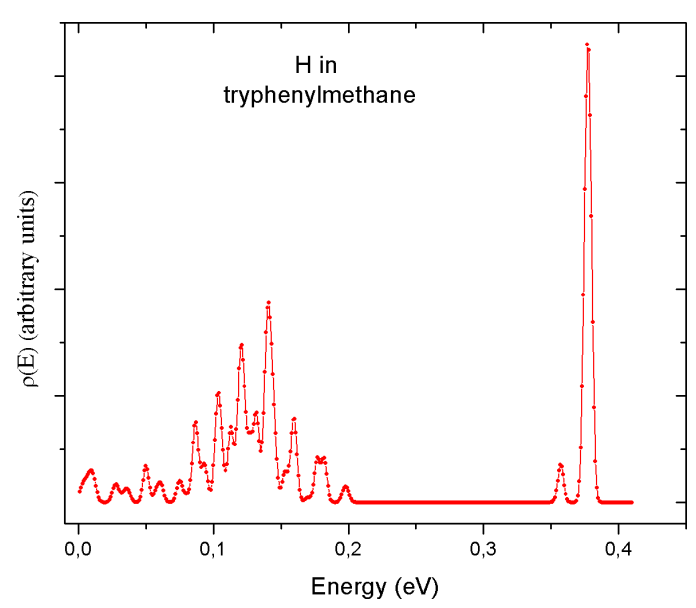

Figure 1. $\mathrm{H}$ in solid- $\mathrm{C}_{19} \mathrm{H}_{16}$ generated by means of the VASP package along with the Phonopy code.

in solid crystals. In phonon calculations using DFT, a description of the vibrational properties of a system only requires knowledge of one fundamental quantity, the forceconstant matrix, or its reciprocal space representation, the dynamical matrix [7].

With this techniques, all internal forces can be calculated when some element is displaced from its equilibrium positions [8]. Once the dynamical matrix is known, the harmonic approximation is used to calculate the vibrational modes and its corresponding frequencies. In principle, $3 \mathrm{~N}$ atom displacements are necessary (where $\mathrm{N}$ is the number of atoms in the unit cell) to calculate the vibrational modes. However, this number can be reduced by means of crystal symmetries.

Solid triphenylmethane has $P n a 2_{1}$ /orthorhombic symmetry, containing 8 chemical formula unit inside the unit cell. The lattice parameters are $a=25.491 \AA ; b=14.586 \AA$ and $\mathrm{c}=7.400 \AA[9]$. Forces among atoms were calculated by means of the VASP code [10] which is a DFT implementation, while the $\rho(\varepsilon)$ was obtained in the harmonic approximation by Phonopy code [11]. Energy cutoff of $550 \mathrm{eV}$ was set for the plane wave expansion, along with a mesh of $2 \times 3 \times 4$ k-points in reciprocal space. The GGA approximation was used for the exchange-correlation potential. In order to determine the equilibrium state of the atoms in the unit cell, both, lattice parameters and internal positions were fully relaxed until the forces were smaller than $10^{-4} \mathrm{eV} / \AA$. The supercell approach was use to obtain the forces originated by the small displacements, which in our case was defined with 16 unit formula (560 atoms)[11]. The calculation of the forces for 1680 different displacements in the supercell, was reduced to 420 due to the symmetries of the solid.

Finally, to obtain $\rho(\varepsilon)$, the eigenvalue problem of dynamical matrix in reciprocal space representation was solved for each q-point in a mesh of $3 \times 6 \times 9$ [11]. The resulting frequency spectrum for $\mathrm{H}$ in solid triphenylmethane is shown in Figure 1. This spectrum was used by the LEAPR module of NJOY in order to generate the cross section library of $\mathrm{H}$ in $\mathrm{C}_{19} \mathrm{H}_{16}$.

\section{Experimental results on triphenylmethane cross-section}

A set of neutron transmission experiments with a triphenylmethane sample were carried out on the VESUVIO spectrometer at the ISIS Neutron and Muon Source (United Kingdom). ISIS is a short-pulse spallation neutron source where neutrons are produced through interactions of an $800 \mathrm{MeV}$ proton beam with tantalum-clad tungsten targets within Target Station 1 (TS1) and Target Station 2 (TS2). The Proton-beam pulse has a repetition rate of 50 $\mathrm{Hz}$. In every set of five pulses, the first four (with a period of $20 \mathrm{~ms}$ per pulse) go to TS1 and the fifth to TS2. The VESUVIO spectrometer [12] is located at TS1 looking at a water moderator. With this instrument it is also possible to measure the total cross section of a sample material, by the transmission and time of flight techniques, over five decades in energy $\left(10^{-3}\right.$ to $\left.10^{2} \mathrm{eV}\right)$. In Figure 2 the general setup of VESUVIO is shown [13]. The transmission monitor $\mathrm{S} 2$ is a ${ }^{6} \mathrm{Li}$-doped glass scintillator placed at $13.43 \mathrm{~m}$ from the neutron moderator. The beam monitor $\mathrm{S} 1$ (also a ${ }^{6} \mathrm{Li}$ scintillator) is placed upstream the sample position at $8.60 \mathrm{~m}$ from the moderator, and its count rate is employed to normalize the spectra registered by the transmission monitor. The cryostat with the sample was placed at $11 \mathrm{~m}$ from the moderator.

A sample of triphenylmethane powder was purchased from Santa Cruz Biotechnology. Two $0.5 \mathrm{~mm}$ thick, square shape $\left(90 \times 90 \mathrm{~mm}^{2}\right)$ aluminum containers were used for the experiments (see Figure 3). In order to get a homogenous sample, one of the mentioned containers was filled by compressing the powder with a spatula and with the top of the container. As the melting point of the triphenylmethane is $365.6 \mathrm{~K}$, we heated the sample to make it liquid and to get, after cooling it down, a solid homogeneous sample of constant thickness. After doing that, the container was closed using 16 screws, nuts and washers. The mass inside the container was $10.64 \mathrm{~g}$ with $0.1 \%$ of confidence. After filling the sample, an identical empty container was prepared for transmission measurements. In both, a heater and a sensor were placed at the same positions.

Transmission measurements were performed on triphenylmethane at four different temperatures: $22 \mathrm{~K}, 50 \mathrm{~K}$, $100 \mathrm{~K}$ and $150 \mathrm{~K}$. All these measurements were carried out using the same cryostat. Spectra were recorded as a function of the time-of-flight, and converted to energy employing programs based on the Open Genie display and data analysis suite, developed for the neutron scattering instruments at the ISIS facility [14]. The sample transmissions were determined by measuring the transmitted beam $S(\varepsilon)$, and the empty container $C(\varepsilon)$.

The background $B(\varepsilon)$ was measured by using a sample which was built with $5 \mathrm{~mm}$ polyethylene plus 2 cadmium sheets of $0.5 \mathrm{~mm}$ each. The measurement results confirmed that the background contribution can be neglected in the energy range where our measurements have been performed. The similar conclusion was drawn in the analysis of the mean emission time corrections, they were 


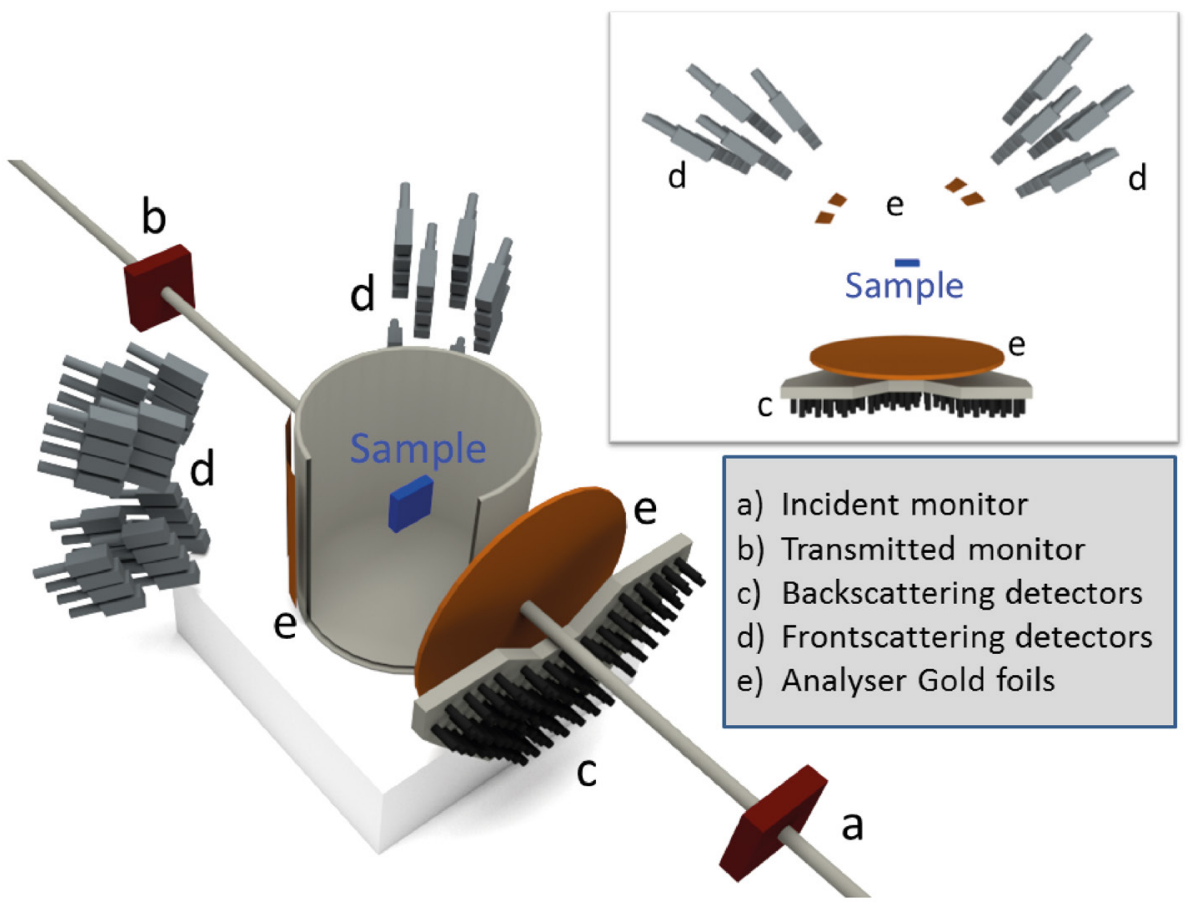

Figure 2. VESUVIO general setup, showing the forward scattering and backscattering detectors [13].

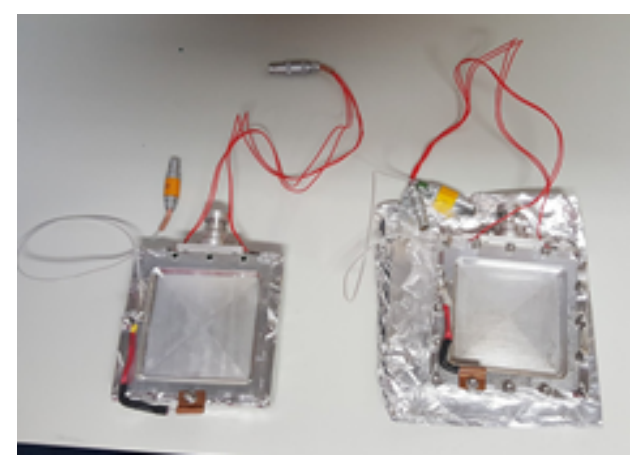

Figure 3. Two identical sample-containers prepared for $\mathrm{C}_{19} \mathrm{H}_{16}$ sample and empty container measurements.

calculated based on the neutron moderator material and proved to be irrelevant for the present case.

With these considerations, the transmission which is defined as $T(\varepsilon)=\frac{S(\varepsilon)-B(\varepsilon)}{C(\varepsilon)-B(\varepsilon)}$ is related to the total cross section $\sigma_{\text {tot }}(\varepsilon)$ via

$$
T(E)=e^{-n \cdot d \cdot \sigma_{\text {tot }}(\varepsilon)}
$$

where $n$ is the number density and $d$ is the sample thickness. Both $n$ and $\sigma_{\text {tot }}$ are determined per molecule. Thus, the cross section can be calculated as:

$$
\sigma_{\text {tot }}(\varepsilon)=-\frac{1}{n \cdot d} \ln (T(\varepsilon))
$$

In Figure 4 the experimental total scattering cross section for the triphenylmethane are shown at the four temperatures. It can be seen that the cross section is practically independent of temperature above $30 \mathrm{meV}$. As expected, between 0.3 and $30 \mathrm{meV}$ the cross-section increases with increasing temperature.

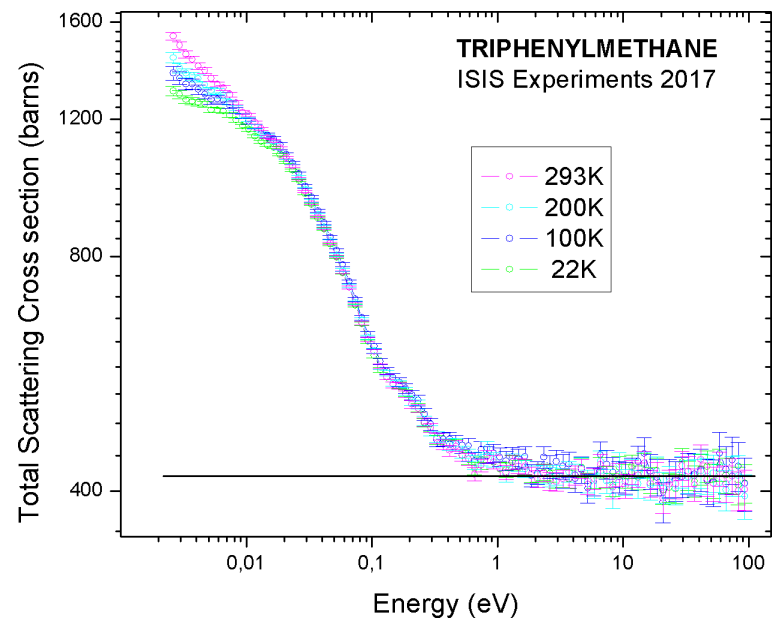

Figure 4. Experimental total scattering cross section for triphenylmethane obtained at VESUVIO spectrometer by neutron transmission technique.

In Figure 5 experimental and calculated total scattering cross section for $\mathrm{H}$ bound in tryphenylmethane are shown for $22 \mathrm{~K}$ and $293 \mathrm{~K}$. Good agreement is found.

\section{Conclusions}

We present a new model for the generation of the scattering kernels for triphenylmethane, together with experimental results on its total cross section measured at the VESUVIO instrument (ISIS Neutron and Muon Source, United Kingdom). The thermal scattering kernel was generated using the NJOY Nuclear Data Processing system with a model based on calculations performed using the 

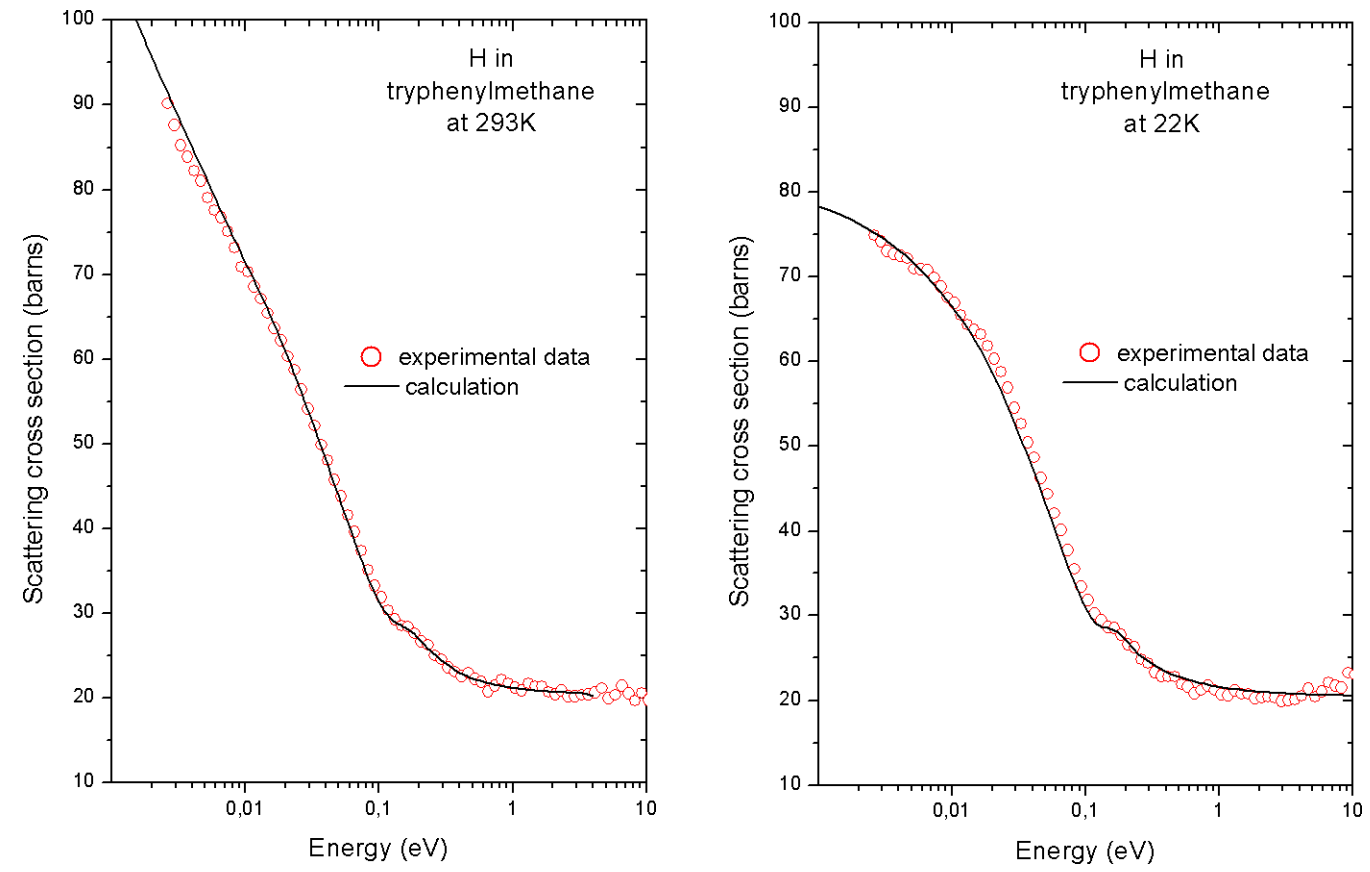

Figure 5. Comparison between experimental data and our model prediction for $\mathrm{H}$ in $C_{19} H_{16}$ at $22 \mathrm{~K}$ and $293 \mathrm{~K}$.

harmonic approximation and forces calculated by density functional theory. The very good agreement between measurements and our model results has been found. This validates the scattering kernel construction and the cross section library produced in ENDF and ACE formats.

\section{Acknowledgments}

The authors are grateful to the UK Science and Technologies Facilities Council for the beam time allocated. Also C.H. used HPC resources from D.C.A.P.- G.T.I.C.C.N.E.A. URL: http://www.cnea.gov.ar/

\section{References}

[1] F. Cantargi, J.R. Granada, NIMB 268 (2010) 24872491.

[2] G.L. Squires, Thermal Neutron Scattering, Cambridge University Press (1978).

[3] J.R. Granada et al., IAEA CRP 1916 / F1.20.26 "Advanced Moderators for Intense Cold Neutron Beams in Materials Research", Vienna, 22-26 Feb (2016).

[4] F. Cantargi, J.R. Granada, J.I. Márquez Damián, EPJ Web of Conferences 146, 13003 (2017).

[5] R.E. MacFarlane, D.W. Muir, The NJOY Nuclear
Data processing System, Los Alamos National Laboratory Report LA-12740-M (1994).

[6] J.I. Márquez Damián et al., Nuclear Data Sheets 118 (2014) 169.

[7] C. Andreani, M. Krzystyniak, G. Romanelli, R. Senesi, F. Fernandez-Alonso, Electron-volt neutron spectroscopy: beyond fundamental systems, Advances in Physics, DOI: 10.1080/00018732.2017.1317963 (2017) [8] R. Martin, Electronic Structure: basic theory and practical methods. ISBN: 0-52-178285-6.

[9] N. Veldman, A. Spek, J.J.H.Schlotter, J.W. Zwikker, L.W. Jenneskens, Acta Crystallographica Section C, 52, (1996), 174-177.

[10] G. Kresse and J. Hafner, J. Phys.: Condens. Matt. 6, 8245 (1994).

[11] A. Togo, L. Chaput, I. Tanaka, G. Hug, Phys. Rev. B, 81, 174301-1-6 (2010).

[12] C. Andreani, D. Colognesi, J. Mayers, G.F. Reiter, R. Senesi, Adv. Phys. 54 (2005) 377.

[13]https://www.isis.stfc.ac.uk/Pages/Vesuvio.aspx https://www.isis.stfc.ac.uk/Pages/Vesuvio.aspx

[14] F.A. Akeroyd, R.L. Ashworth, S.D. Johnston, J.M. Martin, C.M. Moreton-Smith, D.S. Sivia, Open GENIE User Manual, Tech. rep. Rutherford - Appleton: Laboratory Technical Report RAL-TR-2000-002, 2000. 